\title{
Reversal of experimental diabetic neuropathy by VEGF gene transfer
}

\author{
Peter Schratzberger, ${ }^{1}$ Dirk H. Walter, ${ }^{1}$ Kilian Rittig, ${ }^{1}$ Ferdinand H. Bahlmann, ${ }^{1}$ \\ Roberto Pola, ${ }^{1}$ Cynthia Curry, ${ }^{1}$ Marcy Silver, ${ }^{1}$ Joseph G. Krainin, ${ }^{1}$ David H. Weinberg, ${ }^{2}$ \\ Allan H. Ropper, ${ }^{2}$ and Jeffrey M. Isner ${ }^{1,3}$ \\ ${ }^{1}$ Division of Cardiovascular Research, \\ ${ }^{2}$ Division of Neurology, and \\ ${ }^{3}$ Division of Vascular Medicine, Department of Medicine, St. Elizabeth's Medical Center, Tufts University School of Medicine, \\ Boston, Massachusetts, USA
}

Address correspondence to: Jeffrey M. Isner, St. Elizabeth's Medical Center, 736 Cambridge Street, Boston, Massachusetts 02135, USA. Phone: (617) 789-2392; Fax: (617) 779-6362; E-mail:VeJeff@aol.com.

Received for publication January 10, 2001, and accepted in revised form March 19, 2001.

The pathogenetic basis for diabetic neuropathy has been enigmatic. Using two different animal models of diabetes, we have investigated the hypothesis that experimental diabetic neuropathy results from destruction of the vasa nervorum and can be reversed by administration of an angiogenic growth factor. Nerve blood flow, as measured by laser Doppler imaging or direct detection of a locally administered fluorescent lectin analogue, was markedly attenuated in rats with streptozotocin-induced diabetes, consistent with a profound reduction in the number of vessels observed. A severe peripheral neuropathy developed in parallel, characterized by significant slowing of motor and sensory nerve conduction velocities, compared with nondiabetic control animals. In contrast, 4 weeks after intramuscular gene transfer of plasmid DNA encoding VEGF-1 or VEGF-2, vascularity and blood flow in the nerves of treated animals were similar to those of nondiabetic control rats; constitutive overexpression of both transgenes resulted in restoration of large and small fiber peripheral nerve function. Similar experiments performed in a rabbit model of alloxan-induced diabetes produced comparable results. These findings support the notion that diabetic neuropathy results from microvascular ischemia involving the vasa nervorum and suggest the feasibility of a novel treatment strategy for patients in whom peripheral neuropathy constitutes a secondary complication of diabetes.

J. Clin. Invest. 107:1083-1092 (2001).

\section{Introduction}

Peripheral neuropathy is a frequent complication of diabetes that ultimately accounts for significant morbidity. Symptoms are typically dominated by sensory defects (1). The ultimate consequence of such sensory deficits involving the lower extremities may be foot ulceration initiated by traumatic injury that is inapparent to the patient. Indeed, it has been reported that $20 \%$ of all hospital admissions among diabetic patients in the US are for foot problems (2). That such ulcerations may lead to lower extremity amputation (3) is borne out by the fact that the rate of lower limb amputation is 15 times higher in diabetic than in nondiabetic patients (4). Even with intensive insulin therapy, as reported in the Diabetes Control and Complications Trial (5), the incidence of new clinically detected neuropathy per patient-year was as high as $7.0 \%$; with conventional therapy, the incidence of neuropathy increased to as much as $16.1 \%$ (5).

When loss of sensation is compounded by loss of control over blood flow due to autonomic neuropathy and lower extremity vascular obstruction, the threat of limb loss is exacerbated. In the case of peripheral artery disease, hospital mortality, length of hospitalization, and complications resulting from surgery are all increased in the presence of diabetes (6).

Diabetic polyneuropathy has been postulated to occur by diverse pathogenetic mechanisms; modification and inactivation of proteins critical to neural function by nonenzymatic glycosylation (7), altered neural polyol metabolism $(8,9)$, reduced availability of neurotrophic factors $(1,10)$, and microvascular disease with impaired blood flow (11-13) and ischemia in diabetic nerves $(14,15)$ have all been implicated in the pathogenesis of diabetic complications.

Accordingly, we investigated the hypothesis that experimental diabetic neuropathy results from destruction of the vasa nervorum and can be reversed by administration of VEGF $(16,17)$, an endothelial cell mitogen that has been previously shown to promote angiogenesis in a variety of animal models (18-21) and, more recently, in human subjects (22-25).

We document that the disordered peripheral nerve physiology resulting from experimentally induced diabetes is associated with marked destruction of the vasa nervorum of the sciatic nerve and that both the neuropathy and loss of vasa nervorum may be successfully modified by VEGF gene transfer. 


\section{Methods}

Animal models

All protocols were approved by St. Elizabeth's Institutional Animal Care and Use Committee. In all experiments, investigators performing the follow-up examinations were blinded to the identity of the treatment administered.

\section{Rats}

Female Sprague-Dawley rats (Charles River Laboratories, Wilmington, Massachusetts, USA) weighing 200-250 g were used. Rats were fed standard lab rodent chow and water ad libitum and housed individually.

Induction of diabetes. Diabetes was induced after an overnight fast with a single intraperitoneal injection of streptozotocin $(50 \mathrm{mg} / \mathrm{kg}$ in $0.9 \%$ sterile saline $)$ into anesthetized rats $(5 \mathrm{mg} / 100 \mathrm{~g}$ pentobarbital, intraperitoneally). Rats were provided $10 \%$ dextrose solution ad libitum overnight, following which they were returned to standard laboratory chow and to water ad libitum. Serum glucose levels were measured 1 week later, and all animals with levels less than $400 \mathrm{mg} / \mathrm{dl}$ were excluded from these studies. Serum glucose levels were checked again before gene transfer, and any animals with serum glucose levels less than $400 \mathrm{mg} / \mathrm{dl}$ were discarded. Because this dose of streptozotocin causes subtotal destruction of pancreatic $\beta$ cells, the rats survive without exogenous insulin. Age- and weightmatched rats were used as nondiabetic control animals.

\section{Rabbits}

New Zealand white rabbits (Pine Acre Rabbitry, Norton, Massachusetts, USA) weighing 3.5-4.0 kg were used for these experiments. Rabbits were fed standard laboratory rabbit chow (417 kcal/100 g); water was provided ad libitum; and rats were housed individually.

Induction of diabetes. Rabbits were rendered diabetic by treatment with alloxan $(100 \mathrm{mg} / \mathrm{kg}$ of body weight; Sigma Chemical Co., St. Louis, Missouri, USA) dissolved in $4 \mathrm{ml} \mathrm{NaCl}(0.15 \mathrm{M})$ and immediately administered intravenously to the anesthetized rabbit via the marginal ear vein. A total of $10 \mathrm{ml}$ glucose $(50 \% \mathrm{wt} / \mathrm{vol})$ was administered subcutaneously 4,8 , and 12 hours after injection to offset transient hypoglycemia developing after alloxan treatment. Rabbits were considered diabetic when fasting plasma glucose concentrations were greater than $300 \mathrm{mg} / \mathrm{dl}$. Because this dose of alloxan causes total destruction of pancreatic $\beta$ cells, daily insulin (NPH; Novo Nordisk, Princeton, New Jersey, USA) is required to maintain plasma glucose levels between 300 and 400 mg/dl. Age- and weight-matched rabbits were used as nondiabetic control animals.

\section{VEGF plasmids and gene transfer}

phVEGF $F_{165}$. Complementary DNA clones for recombinant human VEGF $_{165}$, isolated from cDNA libraries prepared from HL-60 leukemia cells, were assembled into a simple eukaryotic expression plasmid that uses the 736-bp cytomegalovirus promoter/enhancer to drive VEGF expression. Downstream from the VEGF cDNA is an SV40 polyadenylation sequence. Also included in this plasmid is a fragment containing the SV40 origin of replication that includes the 72-bp repeat, but this sequence is not functionally relevant in the absence of the SV40 T antigen. These fragments occur in the pUC118 vector, which includes an Escherichia coli origin of replication and the $\beta$-lactamase gene for ampicillin resistance. The biologic activity of VEGF secreted from cells transfected with this construct ( $\mathrm{phVEGF} \mathrm{F}_{165}$ ) has been confirmed previously by evidence that media conditioned by transfected human 293 cells promoted the proliferation of capillary endothelial cells.

phVEGF-2. The plasmid phVEGF-2 is a 5,283-bp plasmid that contains the human VEGF-2 coding sequence. Expression from the VEGF-2 gene is modulated by the presence of enhancer sequences from cytomegalovirus and promoter sequences of the Rous sarcoma virus. RNA processing signals (rat pre-proinsulin polyadenylation and $3^{\prime}$ splice sequences) are present to enhance VEGF-2 messenger RNA stability. The plasmid also contains a gene that confers kanamycin resistance to the host cells.

Gene transfer. Bilateral intramuscular DNA injections were performed 12 and 26 weeks after the induction of diabetes in rats and rabbits, respectively. After completion of baseline nerve conduction measurements, animals received intramuscular injections into each the femoral quadriceps muscle and the femoral biceps muscle using a 27-gauge needle advanced through small skin incisions. Based on previous studies of VEGF gene transfer in animal models of hindlimb ischemia, diabetic rats received a dose of $250 \mu \mathrm{g}$ of the naked plasmid DNA encoding for the 165-amino acid isoform of human VEGF-1 (phVEGF $165,125 \mu \mathrm{g} / 0.5 \mathrm{ml}$ sterile saline per limb; ref. 26) or $1,000 \mu \mathrm{g}$ of the naked plasmid DNA encoding for human VEGF-2 (phVEGF-2, 500 $\mu \mathrm{g} / 0.5 \mathrm{ml}$ per side; ref. 27$)$. Diabetic rabbits received bilateral injections of phVEGF-2 at a total dose of 1,000 $\mu \mathrm{g}$ in $10 \mathrm{ml}$ of saline per limb. From a functional standpoint, VEGF-2 has been shown to be similar to VEGF 165 with regard to its ability to promote angiogenesis, both in laboratory animals (27) and, in more recent trials, in human subjects (J. Isner, unpublished data). Like $V_{E G F}{ }_{165}, V E G F-2$ ligates the KDR receptor (VEGF receptor-2), but in addition, it ligates the Flt-4 receptor (VEGF receptor-3; ref. 28).

\section{Neurophysiological measurements}

Electrophysiology. Nerve conduction velocity was measured bilaterally using sciatic nerve motor $(M)$ and sensory $(\mathrm{H})$ waves $(29)$ in all rats and rabbits at baseline (before treatment) and then at 2, 4, and 10 weeks after treatment in rats, and at 4 and 8 weeks after treatment in rabbits. Results from both sides were averaged. Sciatic nerves were stimulated at two sites using paired percutaneous monopolar needle electrodes as described 
elsewhere (29); the proximal stimulation site was the sciatic notch, and the distal stimulation site was at the ankle. A surface electrode was placed at the base of the tail to serve as a ground. Supramaximal square wave stimulations of fixed duration (0.1 millisecond) (Teca TD-10; Oxford Instruments, Pleasantville, New York, USA) were applied to the nerve, and the resulting muscle compound action potentials (CMAPs) were recorded from the interosseous muscles of the ipsilateral foot with two needle electrodes. The mean latency difference between three pairs of the distal and proximal CMAP responses was determined and combined for each data point. Motor nerve conduction velocity (MCV; meters per second) was calculated by dividing the interelectrode distance between the two stimulation sites (in millimeters) as measured with calipers by the mean latency difference (in milliseconds) of the CMAPs. The sensory nerve conduction velocity (SCV) was determined similarly, using the same stimulating and recording electrode pairs by measuring the latency difference of the H-reflex, a monosynaptic spinal loop in which stimulation of afferent proprioceptive Ia fibers excite segmental spinal cord $\alpha$-motor neurons (30). Six pairs of notch- and ankle-evoked $\mathrm{H}$-wave reflexes were recorded and averaged.

Tailflick testing. This behavioral test examined the response of each rat to a thermal noxious stimulus administered to its tail using a variation of the tail immersion test. This measure was chosen because of the role small fiber dorsal root ganglia sensory neurons play in pain transmission. The animals were loosely restrained with their tails immersed in a beaker of water to a depth of about $2 \mathrm{~cm}$. The beaker was uni- formly heated at a rate of about $2{ }^{\circ} \mathrm{C}$ per minute beginning at $35^{\circ} \mathrm{C}$. A magnetic stirring bar was used to maintain a uniform temperature. The temperature at which each animal flicked its tail out of the water was recorded to the nearest $0.5^{\circ} \mathrm{C}$.

\section{Assessment of transgene expression}

VEGF protein expression in tissue. Sciatic nerves and samples from the femoral quadriceps and the femoral biceps muscles from rats and rabbits were harvested 2 weeks after VEGF-1 and VEGF-2 gene transfer, respectively. Samples were homogenized in lysis buffer (100 $\mathrm{mM}$ potassium phosphate, $0.2 \%$ Triton $\mathrm{X}-100$ ) supplemented with a protease inhibitor cocktail (Roche Molecular Biochemicals, Mannheim, Germany). Total protein extracts were quantified by the BCA protein assay kit (Pierce Chemical Co., Rockford, Illinois, USA). Nonreduced or reduced protein extracts $(100 \mu \mathrm{g}$ per sample) were separated on $15 \%$ polyacrylamide gels (Bio-Rad Laboratories Inc., Rockville Center, New York, USA) and electroblotted on a nitrocellulose membrane, which was blocked with $5 \%$ nonfat dry milk in $0.2 \%$ Tween PBS (T-PBS). Samples from VEGF-1-transfected rats were then probed with VEGF goat polyclonal antibody (1:1,000 dilution; Sigma Chemical Co.), and samples from VEGF-2-transfected rabbits were probed with a VEGF-C goat polyclonal antibody (1:2,500; Santa Cruz Biotechnology Inc., Santa Cruz, California, USA) for 2 hours at room temperature. The membrane was washed three times in T-PBS and then incubated with anti-goat $(1: 10,000)$ horseradish peroxidase $\operatorname{IgG}$ for 1 hour. Antigen-antibody complexes were visualized after incubation for 1 minute with enhanced lumines- a

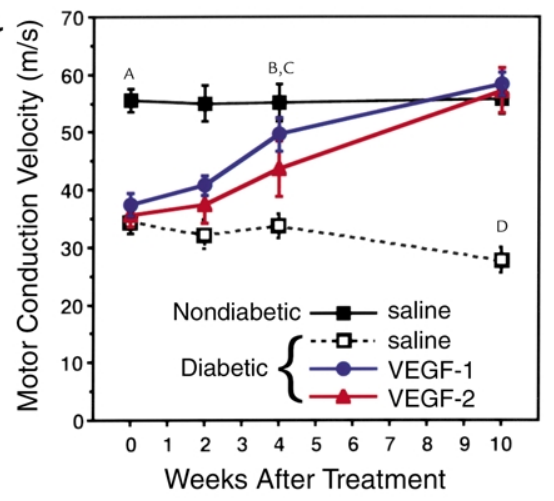

b

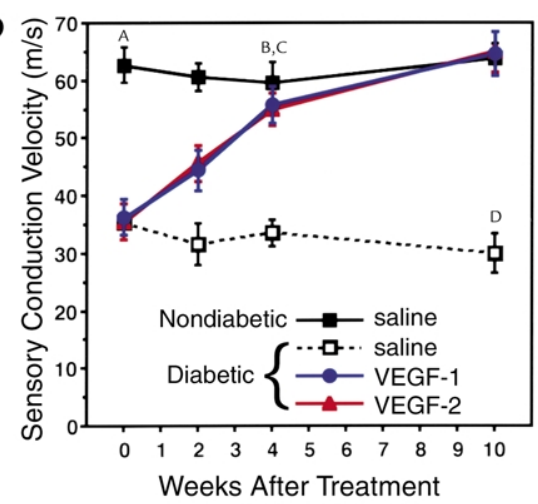

C

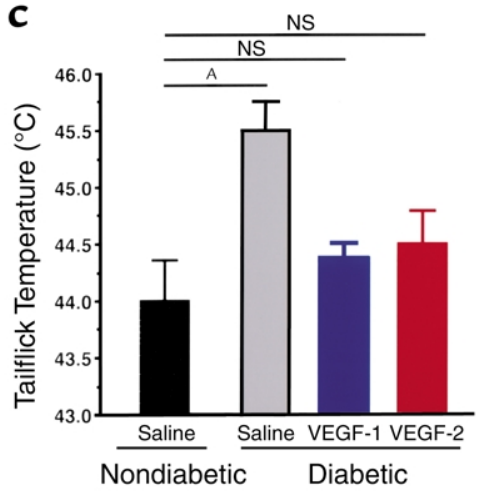

\section{Figure 1}

Time course of MCV (a) and SCV (b). Female Sprague-Dawley rats weighing 240-260 g were injected intraperitoneally with streptozotocin at $50 \mathrm{mg} / \mathrm{kg}$. Diabetes was confirmed by testing blood glucose the next day. Twelve weeks later, nondiabetic and diabetic rats were randomly assigned to a saline-injection control group, and to the treatment groups that underwent either VEGF-1 or VEGF-2 gene transfer (nondiabetic, saline injection, $n=5$; nondiabetic, VEGF-1, $n=6$; nondiabetic, VEGF-2, $n=5$; diabetic, saline-injection, $n=9$; diabetic, VEGF-1, $n=18$; and diabetic, VEGF-2, $n=15$ ). Sciatic nerve conduction measurements were performed at the time of treatment (week 0$)$ and then at 2, 4, and 10 weeks. Data are expressed as mean \pm SEM. ${ }^{A} P<0.01$, nondiabetic saline versus diabetic saline, diabetic VEGF-1, or diabetic VEGF-2. ${ }^{B}$ Diabetic VEGF-1 or diabetic VEGF-2 not significant versus nondiabetic saline. ${ }^{C} P<0.01$, diabetic saline versus diabetic VEGF-1, diabetic VEGF-2, or nondiabetic. ${ }^{D} P<0.01$, diabetic saline versus diabetic VEGF-1, diabetic VEGF-2, or nondiabetic. (c) Tailflick threshold temperatures 4 weeks after gene transfer. The temperature at which the rats demonstrated the characteristic tailflick response was tested for in a completely blinded fashion. Values are expressed as mean \pm SEM. ${ }^{A} P<0.01$. Nondiabetic, saline-injection, $n=5$; diabetic, salineinjection, $n=9$; diabetic, VEGF-1, $n=18$; and diabetic, VEGF-2, $n=15$. 


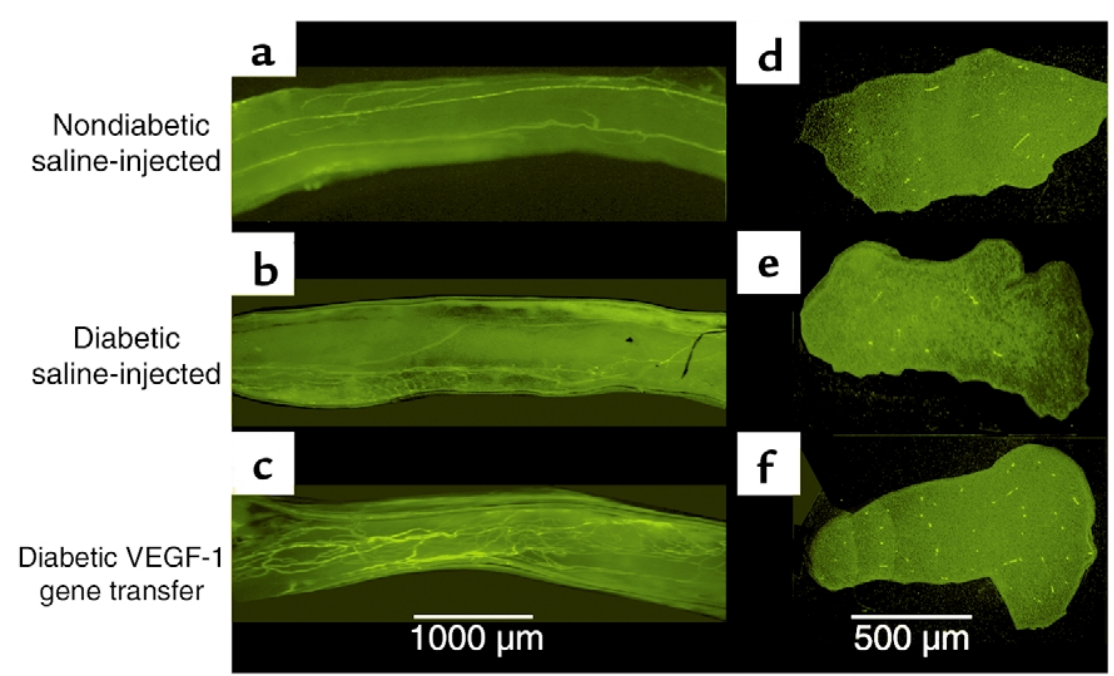

Figure 2

Representative fluorescence photomicrographs of longitudinal views of whole-mounted rat sciatic nerves (a-c) and their respective paraffin-embedded cross sections (d-f) 4 weeks after treatment. Before sacrifice and harvesting of the nerves, in vivo perfusion with FITC-conjugated BS-1 lectin, an endothelial-specific ligand, was performed. (a and d) Samples taken from a nondiabetic saline-injected control animal, showing a normal pattern of vascularity. (b and e) Samples taken from a diabetic animal 12 weeks after induction of diabetes and 4 weeks after sham treatment (saline injection). The total network of vasa nervorum is markedly reduced, resulting in an irregular distribution pattern and areas of nonvascularized nerve tissue. Note in particular the reduction of stained endoneurial vessels in the cross-sectional image. ( $\mathbf{c}$ and $\mathbf{f}$ ) Samples from a rat after 12 weeks of diabetes and 4 weeks after VEGF-1 gene transfer. Vascularity appears well preserved, and the number of visible vessels in the cross section appears similar to that of a normal sciatic nerve. $\times 2(\mathbf{a}-\mathbf{c}) ; \times 4(\mathbf{d}-\mathbf{f})$.

cence reagent (Amersham Pharmacia Biotech, Piscataway, New Jersey, USA) at room temperature, followed by exposure to hyperfilms (Amersham Pharmacia Biotech). Equal protein loading among individual lanes was confirmed after stripping the membranes with ImmunoPure elution buffer (Pierce Chemical Co.) by reprobing the membranes with an $\alpha$-tubulin mouse mAb (1:1,000 dilution; Calbiochem-Novabiochem Corp., San Diego, California, USA). Each experiment was repeated at least three times with different cellular extracts. Densitometric analysis was performed (NIH Image 1.62; Research Service Branch of the National Institute of Mental Health) to enable quantitative comparison of protein expression. Results shown are representative of three to five experiments.

In vivo assessment of perfusion and vascularity

Laser Doppler imaging of vasa nervorum blood flow. Perfusion of sciatic vasa nervorum was measured bilaterally in hindlimbs of rats and rabbits, respectively, with a laser Doppler perfusion imager (LDPI) system (Moor Instruments, Wilmington, Delaware, USA) (20, 31), programmed to measure perfusion of surrounding tissue as zero, or background. After anesthesia and completion of electrophysiological testing, nerves were exposed by scalpel incision and blunt dissection of the overlying muscle and connective tissue fascia. Care was taken not to disturb the sciatic nerve and its vascula- ture. Immediately after exposure of the nerves, measurements were repeated three times over the same region of interest. The perfusion signal was split into six different intervals, each displayed in a separate color. Low or no perfusion was displayed in dark blue, whereas the maximum perfusion interval was displayed in red. Colorcoded images were recorded, and analyses were performed by calculating the average perfusion for each nerve. All perfusion measurements, as well as neurophysiological examinations (see below), were carried out with the animal placed on a heating blanket underneath a warming lamp. Both blanket and lamp were connected to a thermostat set at $37^{\circ} \mathrm{C}$.

Fluorescent imaging of vasa nervorum. Vascularity of sciatic nerves from both normal and diabetic rats and rabbits was assessed by in situ fluorescent staining using the endothelial cell-specific marker BS-1 lectin conjugated to FITC (Vector Laboratories, Burlingame, California, USA) $(32,33)$. After anesthesia, the aorta was catheterized via a laparotomy, and the hindlimbs were perfused with BS-1 lectin (1 mg in rats, $5 \mathrm{mg}$ in rabbits), and both iliac veins were ligated to facilitate in situ incubation of the lectin-FITC conjugate. Fifteen minutes later, the animals were sacrificed and the nerves (rat: sciatic; rabbit: tibial portion of sciatic nerves) were harvested and

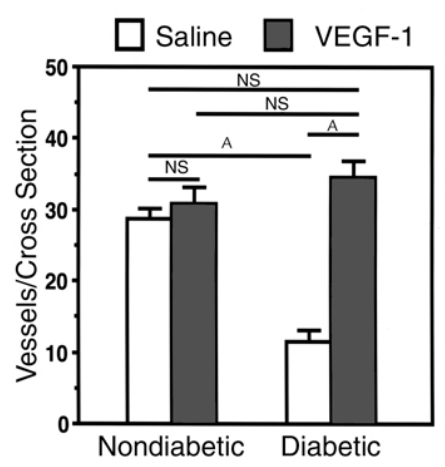

Figure 3

Quantification of vessels in tissue cross sections of rat sciatic nerve. Before sacrifice at 4 weeks after treatment (gene transfer or saline injections), animals were perfused with FITC-conjugated BS-1 lectin to visualize vasa nervorum. Ten cross sections per paraffin-embedded specimen were randomly selected from each specimen, and vessels per cross section were counted under a fluorescence microscope $(x 4)$ by an investigator blinded to treatment group. Data are expressed as mean \pm SEM ( $n=5$ per study group), where individual numbers per animal were averaged from right and left sciatic nerves. ${ }^{A} P<0.01$. 
a

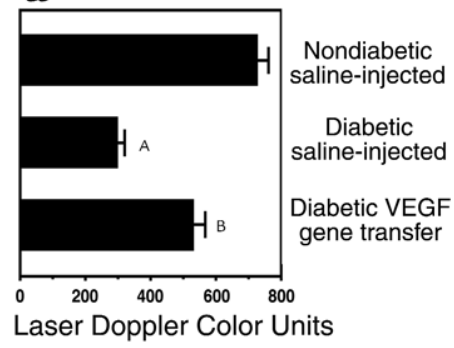

b

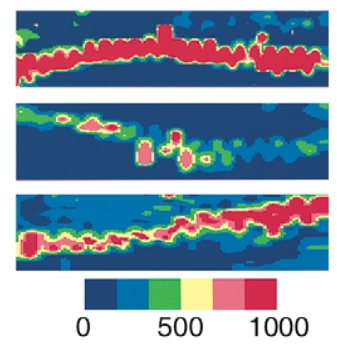

Laser Doppler Color Units

\section{Figure 4}

In vivo LDPI of blood flow in rat sciatic nerve 4 weeks after gene transfer or saline injection. Nerves were surgically exposed from the sciatic notch to the knee level before three repeated LDPI measurements were obtained from the region of interest. (a) Bar graph (mean \pm SEM) summarizes results of LDPI measurements taken from both sides of five rats per study group. ${ }^{A} P<0.01$ versus nondiabetic salineinjected. ${ }^{B} P<0.01$ versus diabetic saline-injected. (b) Representative color-coded LDPI. Lowest blood flow is indicated in blue, maximum blood flow in red, and intermediate grading in green and yellow. Nondiabetic saline-injected: perfusion of sciatic nerve in a normal, age-matched rat that underwent saline injection. Diabetic salineinjected: markedly reduced perfusion of sciatic nerve in a diabetic rat, 4 weeks after saline injection. Diabetic VEGF gene transfer: substantial restoration of sciatic nerve perfusion in a diabetic rat 4 weeks after VEGF gene transfer.

fixed in $1 \%$ paraformaldehyde for 2 hours. After fixation, nerves were either whole mounted for longitudinal analysis or paraffin embedded for cross-sectioning. Samples were analyzed using a Nikon Diaphot fluorescence microscope (Nikon Inc., Melville, New York, USA) and an Optronics PictureThis digital camera (Optronics Inc., Bolton, Massachusetts, USA).

\section{Renal function}

To assess potential effects of VEGF gene transfer on renal function, rats were housed in metabolic cages for 24 hours, and their total urine volume during that period was collected. Aliquoted samples were later analyzed by standard laboratory methods for their protein and glucose contents. At the same time, blood was taken from these rats and samples were analyzed for creatinine, urea, and glucose by standard laboratory methods. At sacrifice, both kidneys were harvested from each rat, and their weight was corrected for total body weight.

\section{Statistics}

All results are expressed as the mean \pm SEM. Statistical comparisons between groups were performed by ANOVA. $P$ values less than 0.05 were considered statistically significant.

\section{Results}

Studies in rats. Within 12 weeks of the onset of diabetes induced by streptozotocin $(50 \mathrm{mg} / \mathrm{kg})$, rats developed a severe peripheral neuropathy, as described previously (10). Electrophysiological recordings documented significant slowing of MCV and SCV $(\mathrm{MCV}=34.2 \pm 1.7$ $\mathrm{m} / \mathrm{s}$ and $\mathrm{SCV}=35.2 \pm 1.8 \mathrm{~m} / \mathrm{s}$ ), compared with those of nondiabetic control animals $(\mathrm{MCV}=55.5 \pm 2.0 \mathrm{~m} / \mathrm{s}$ and $\mathrm{SCV}=62.6 \pm 3.1 \mathrm{~m} / \mathrm{s} ; P<0.01$ for both MCV and $\mathrm{SCV}$ ) (Figure 1).

To investigate the potential role of microvascular pathology in the development of diabetic neuropathy, we performed in situ fluorescent imaging of the vasa nervorum (Figure 2). Sciatic nerves from BS-1 lectin-perfused nondiabetic rats showed a normal pattern of vascularity, including a superficial longitudinal network and, at intervals, penetrating branches responsible for providing blood flow to the endoneurial vascular network.

In the nerves of diabetic rats, the total number of vasa nervorum was markedly reduced, resulting in an irregular distribution pattern and areas of nonvascularized nerve tissue (Figure 2). Cross sections from these nerves disclosed a significant reduction in the number of vasa nervorum compared with that of nerves from normal control animals $(11.5 \pm 1.5$ and $30.3 \pm 1.2$ vessels per cross section, respectively; $P<0.01$ ) (Figure 3 ).

To investigate whether the extent of peripheral nerve vascularity correlated with augmented perfusion of the vasa nervorum, we used in vivo LDPI. Perfusion in the sciatic nerves of nondiabetic control rats, calculated as the average of measurements performed in both hindlimbs, measured $724 \pm 28 \mathrm{U}$ (arbitrary laser Doppler color units). In contrast, perfusion was significantly reduced in the sciatic nerves of saline-injected diabetic rats $(298 \pm 48 \mathrm{U} ; P<0.01)$ compared with that of nondiabetic (Figure 4).

To determine the impact of growth factor administration intended to preserve vascularity in the affected nerves, serial neurophysiological follow-up examinations were performed at 2,4 , and 10 weeks after intramuscular injection of DNA plasmids encoding either VEGF-1 (phVEGF 165 ), VEGF-2 (phVEGF-2), or saline.
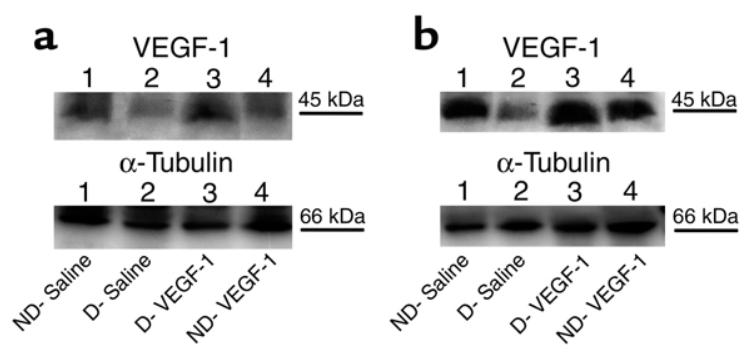

\section{Figure 5}

Expression of VEGF protein in nondiabetic and diabetic (12 weeks after streptozotocin treatment) rats. Western blot analysis of VEGF-1 protein expression in hindlimb muscles (a) and sciatic nerves (b) harvested 2 weeks after sham (saline) or gene injections. The level of VEGF protein expression was significantly reduced in diabetic versus nondiabetic rats. VEGF-1 gene transfer led to restoration of VEGF-1 protein expression both in muscles and in nerves of diabetic rats. Lane 1: nondiabetic saline-injected. Lane 2: diabetic saline-injected. Lane 3: diabetic, VEGF-1 gene transfer. Lane 4: nondiabetic, VEGF-1 gene transfer. VEGF-1 is detected as a $44-k D a$ protein. Western blots were reprobed with an anti- $\alpha-$ tubulin antibody to confirm equal protein loading of lanes. Similar results were obtained in three additional experiments. 
Table 1

Characterization of rats 2 weeks after VEGF-1 gene transfer

\begin{tabular}{lccc}
\hline & $\begin{array}{c}\text { Nondiabetic } \\
\text { saline-injected } \\
(n=4)\end{array}$ & $\begin{array}{c}\text { Diabetic } \\
\text { saline-injected } \\
(n=8)\end{array}$ & $\begin{array}{c}\text { Diabetic VEGF-1 } \\
\text { gene transfer } \\
(n=8)\end{array}$ \\
Body weight $(\mathrm{g})$ & $315.0 \pm 20.0^{\mathrm{A}}$ & $231.7 \pm 22.4$ & $208.3 \pm 10.1^{\mathrm{B}}$ \\
Blood pressure $(\mathrm{mmHg})$ & $119 \pm 7^{\mathrm{A}}$ & $118 \pm 8$ & $115 \pm 8^{\mathrm{B}}$ \\
Heart rate $(\mathrm{bpm})$ & $367 \pm 18^{\mathrm{A}}$ & $344 \pm 19$ & $357 \pm 24^{\mathrm{B}}$ \\
Proteinuria $(\mathrm{mg} / \mathrm{dl})$ & $14.8 \pm 10.5^{\mathrm{A}}$ & $135.7 \pm 7.0$ & $122.7 \pm 52.8^{\mathrm{B}}$ \\
Glucosuria $(\mathrm{mg} / \mathrm{dl})$ & $0.1 \pm 0.0^{\mathrm{A}}$ & $74.6 \pm 11.2$ & $78.5 \pm 22.9^{\mathrm{B}}$ \\
Kidney/body weight ratio & $0.0013 \pm 0.0002^{\mathrm{A}}$ & $0.0023 \pm 0.0004$ & $0.0021 \pm 0.0001^{\mathrm{B}}$ \\
Blood glucose $(\mathrm{mg} / \mathrm{dl})$ & $106.75 \pm 9.2^{\mathrm{A}}$ & $540.2 \pm 33.3$ & $520.9 \pm 22.2^{\mathrm{B}}$ \\
BUN $(\mathrm{mg} / \mathrm{dl})$ & $19.5 \pm 3.8^{\mathrm{A}}$ & $91.7 \pm 16.4$ & $80.0 \pm 13.0^{\mathrm{B}}$ \\
Creatinine $(\mathrm{mg} / \mathrm{dl})$ & $0.5 \pm 0.2^{\mathrm{A}}$ & $0.5 \pm 0.0$ & $0.6 \pm 0.0^{\mathrm{B}}$ \\
& & &
\end{tabular}

Values are expressed as mean \pm SD. ${ }^{A} P<0.05$ versus diabetic saline-injected and diabetic VEGF gene transfer. ${ }^{B}$ Not significant versus diabetic saline-injected (ANOVA).

Intramuscular gene transfer of naked DNA encoding the angiogenic growth factor VEGF-1 or VEGF-2 led to resolution of clinical signs of peripheral neuropathy and of vasculopathy. Saline-injected diabetic rats showed a further deterioration in neuropathy $(\mathrm{MCV}=27.7 \pm 2.2$; $\mathrm{SCV}=30.0 \pm 3.4$ ) (Figure 1 , $\mathrm{a}$ and $\mathrm{b}$ ). In contrast, rats that underwent gene transfer of either VEGF-1 or VEGF-2 demonstrated full restoration of MCV and SCV back to normal levels within 4 weeks after gene transfer. Specifically, MCV in diabetic rats receiving phVEGF-1 was $49.6 \pm 2.8 \mathrm{~m} / \mathrm{s} ; \mathrm{MCV}$ in diabetic rats receiving VEGF-2 was $44.7 \pm 4.7 \mathrm{~m} / \mathrm{s}$; and MCV in nondiabetic rats = $55.1 \pm 3.3 \mathrm{~m} / \mathrm{s}(P=$ not significant [NS] $)$. For SCV, diabetic rats receiving VEGF- 1 measured $55.7 \pm 3.2 \mathrm{~m} / \mathrm{s}$; diabetic rats receiving VEGF-2 measured $54.9 \pm 2.8 \mathrm{~m} / \mathrm{s}$; and nondiabetic rats measured $59.5 \pm 3.7 \mathrm{~m} / \mathrm{s}(P=\mathrm{NS})$ (Figure 1 , $a$ and $b$ ). This improvement persisted for up to 10 weeks after gene transfer. Gene transfer to nondiabetic control animals had no effects on functional nerve parameters (data not shown).

Tailflick testing, a measure of the function of small fiber dorsal root ganglia sensory neurons, was also performed 4 weeks after gene transfer. In saline-injected diabetic rats, tailflick temperatures were significantly increased to $45.5 \pm 0.2^{\circ} \mathrm{C}$. In contrast, tailflick temperatures recorded in diabetic rats from both the VEGF-1 and VEGF-2 treatment groups $\left(44.3 \pm 0.1^{\circ} \mathrm{C}\right.$ and $44.5 \pm 0.3^{\circ} \mathrm{C}$, respectively) did not differ significantly from those of age-matched nondiabetic control animals $\left(44.0 \pm 0.4^{\circ} \mathrm{C}\right)$ (Figure 1).

In diabetic rats with VEGF gene transfer, peripheral nerve vascularity remained well preserved (Figure 2), and cross-sectional images from BS-1 lectin-perfused animals revealed vessel numbers similar to those of nondiabetic control

\section{Table 2} number of rabbits analyzed per group. rats $(34.5 \pm 2.5$ vs. $30.3 \pm 1.2$ vessels per cross section in VEGF-transfected versus nondiabetic rats, respectively; $P=\mathrm{NS}$ ) (Figure 3). Moreover, in diabetic rats undergoing VEGF gene transfer, LDPI showed enhanced perfusion, compared with that of the diabetic saline-injected rats (534 $\pm 44 \mathrm{U} ; P<0.05$; Figure 4).

Transgene expression at the protein level was assessed by Western blotting of nerve and muscle tissue for VEGF. Nerves from diabetic rats contained less VEGF protein than did nerves from nondiabetic control animals, consistent with previous observations from our laboratory (18). The nerves that were harvested from diabetic rats 2 weeks after VEGF gene transfer, however, disclosed VEGF protein expression comparable to that of nondiabetic rats (Figure 5).

To investigate whether VEGF gene transfer could have interfered with glucose metabolism in the study animals, we monitored glucose levels in blood and 24-hour urine samples and found that VEGF-1 and VEGF-2 gene transfer had no effects on either blood or urine glucose levels in nondiabetic or diabetic animals (Table 1). Furthermore, because VEGF may have potentially adverse effects on diabetic nephropathy (34), renal function was monitored in nondiabetic and diabetic rats. We observed that although renal functional parameters were consistent with the development of nephropathy in diabetic animals, VEGF gene transfer did not cause deterioration in preexisting disease, as indicated by similar renal functional parameters and kidney/body weight ratios in both VEGF-transfected and salineinjected control groups. Finally, we also checked body weights, blood pressure, and heart rates of diabetic and nondiabetic animals and found that there were no significant differences between VEGF-transfected and saline-injected animals (Table 1).

Effects of intramuscular VEGF-2 gene transfer on diabetic neuropathy in rabbits

\begin{tabular}{|c|c|c|c|c|c|}
\hline & \multicolumn{3}{|c|}{ Diabetic rabbits } & \multicolumn{2}{|c|}{ Nondiabetic rabbits } \\
\hline & $\begin{array}{c}\text { Saline, } \\
n=5(\mathrm{~m} / \mathrm{s})\end{array}$ & $\begin{array}{c}\text { VEGF-2, } \\
n=14(\mathrm{~m} / \mathrm{s})\end{array}$ & $P$ & $\begin{array}{c}\text { Saline, } \\
n=4(\mathrm{~m} / \mathrm{s})\end{array}$ & $\begin{array}{c}\text { VEGF-2, } \\
n=5(\mathrm{~m} / \mathrm{s})\end{array}$ \\
\hline \multicolumn{6}{|c|}{ Before treatment } \\
\hline MCV & $59.3 \pm 0.8$ & $62.9 \pm 1.3$ & NS & $76.2 \pm 2.6$ & $75.4 \pm 1.2$ \\
\hline SCV & $64.1 \pm 1.1$ & $66.1 \pm 1.8$ & NS & $83.2 \pm 2$ & $80.4 \pm 2.1$ \\
\hline \multicolumn{6}{|c|}{ After 4 weeks } \\
\hline MCV & $58.6 \pm 1.7$ & $68.8 \pm 2.1$ & 0.006 & $73.8 \pm 2.0$ & $76.4 \pm 2$ \\
\hline SCV & $62.5 \pm 4$ & $72.3 \pm 2.2$ & 0.06 & $80 \pm 4.5$ & $82.7 \pm 2$ \\
\hline \multicolumn{6}{|c|}{ After 8 weeks } \\
\hline MCV & $59.6 \pm 2.3$ & $75.2 \pm 2.2$ & 0.001 & $73.8 \pm 2$ & $76.4 \pm 2$ \\
\hline $\mathrm{SCV}$ & $60 \pm 3.2$ & $79.7 \pm 2.8$ & 0.001 & $80 \pm 4.5$ & $82.7 \pm 2$ \\
\hline
\end{tabular}

Six months after induction of diabetes with alloxan $(100 \mathrm{mg} / \mathrm{kg}), \mathrm{MCV}$ and SCV were recorded from both hindlimbs before intramuscular gene transfer of phVEGF-2 encoding for VEGF-2, and 4 and 8 weeks after treatment, by investigators blinded to the treatment regimens. Results from left and right limbs did not differ significantly and were averaged for analysis. Saline injections served as control. Nondiabetic rabbits were age- and weight-matched to diabetic animals. Statistics were calculated using ANOVA; $n$ reflects the total 


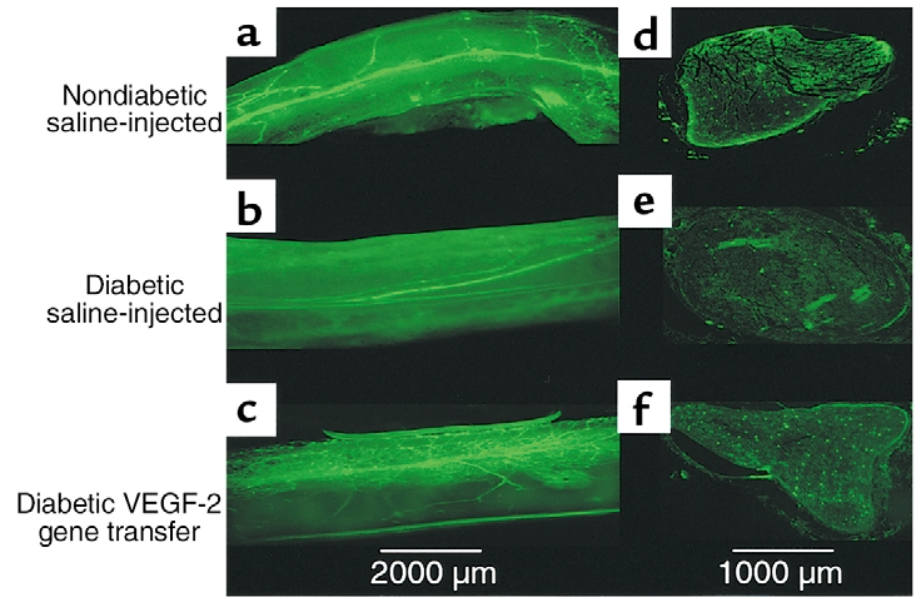

\section{Figure 6}

Representative fluorescence photomicrographs of longitudinal views of whole-mounted rabbit nerves (tibial portion of the sciatic nerve) (a-c) and their respective paraffin-embedded cross sections (d-f) 8 weeks after treatment. Before sacrifice and harvesting of the nerves, in vivo perfusion with FITC-conjugated BS-1 lectin, an endothelial-specific ligand, was performed. ( $\mathbf{a}$ and $\mathbf{d}$ ) Samples from a nondiabetic saline-injected control animal, showing a regular pattern of vascularity. (b and $\mathbf{e}$ ) Samples taken from a diabetic animal 6 months after induction of diabetes and 8 weeks after sham treatment (saline injection). The total network of vasa nervorum is markedly reduced, resulting in an irregular distribution pattern. Note the reduction of stained endoneurial vessels in the cross section. (c and f) Samples from a rabbit after 6 months of diabetes and 8 weeks after VEGF-2 gene transfer. The vascularity appears well preserved, and the number of vessels visible in the cross section appears similar to that of a normal sciatic nerve. $\times 2(\mathbf{a}-\mathbf{c}) ; \times 4(\mathbf{d}-\mathbf{f})$.

Studies in rabbits. To determine whether these findings could be reproduced in an animal model of insulin-dependent diabetes, similar experiments were performed in rabbits with alloxan-induced diabetes. Six months after induction of diabetes, MCV and SCV were both significantly slowed $(\mathrm{MCV}=61.9 \pm 1.0$; $\mathrm{SCV}=65.6 \pm 1.4)$ compared with nondiabetic rabbits $(\mathrm{MCV}=75.8 \pm 1.5 ; \mathrm{SCV}=82.0 \pm 2.0 ; P<0.01)$. Four weeks after VEGF-2 gene transfer, a trend toward improvement of neurological function was apparent. After an additional 4 weeks (8 weeks after VEGF-2 gene transfer), both MCV and SCV were fully restored to levels similar to those found in the age- and weightmatched nondiabetic control rabbits. In diabetic rabbits that received saline injections as placebo, however, no changes in MCV and SCV (Table 2) were observed within the 8 -week observation period.
To investigate pathological alterations of the vasa nervorum in this animal model, and potential angiogenic effects of VEGF-2 gene transfer on the vasa nervorum in diabetic neuropathy, we performed ex vivo fluorescent imaging (Figure 6). In contrast to the normal distribution of vasa nervorum in the sciatic nerves of nondiabetic rabbits, nerves from diabetic rabbits displayed an overall reduction in the number of vessels, with an irregular distribution pattern and areas of nonvascularized nerve tissue. Cross sections from these nerves confirmed the reduced number of vasa nervorum compared with that of nondiabetic controls. After VEGF-2 gene transfer, however, nerves from diabetic rabbits appeared well vascularized, and histological cross sections revealed vessel numbers similar to those of nondiabetic controls (Table 3).

Transfected and nontransfected diabetic and nondiabetic rabbits were also studied in vivo by LDPI to document perfusion of vasa nervorum. Results from these studies disclosed that sciatic nerves from VEGF-2-transfected diabetic rabbits do not only have a greater number of microvessels, but they also show correspondingly augmented perfusion of their vasa nervorum (808 $\pm 60 \mathrm{U} ; n=10)$, whereas sciatic nerve perfusion in untreated diabetic rabbits was severely reduced $(431 \pm 28 \mathrm{U} ; n=12$; $P<0.01$ versus diabetic, VEGF-2-transfected rats). Perfusion for nondiabetic rabbits was $898 \pm 32 \mathrm{U}(P<0.01$ versus diabetic saline-injected rats, and NS versus diabetic VEGF-2-transfected rats) (Figure 7).

To assess the levels of VEGF-2 protein expression and the results of transgene expression in this study, we performed Western blotting for VEGF-2 expression in sciatic nerves and transfected hindlimb muscles. Densitometric analysis of multiple experiments performed on samples from four different animals per group revealed that VEGF-2 protein expression in the sciatic nerves from diabetic animals was reduced to $57.2 \pm 3 \%$ compared with nondiabetic controls (100\%). VEGF-2 gene transfer yielded a restoration of VEGF-2 expression to $92.8 \pm 4.7 \%$ of that found in diabetic rabbits. Similarly, in specimens of muscle samples from diabetic animals, VEGF-2 protein expression was reduced to $22.7 \pm 8 \%$ of that in nondiabetic animals. VEGF-2 gene transfer restored VEGF-2 protein expression to $87.4 \% \pm 4 \%$ of that in nondiabetic animals. Representative results are shown in Figure 8.

Table 3

Quantification of vessels on rabbit tibial nerve cross sections

\footnotetext{
Nondiabetic saline-injected Nondiabetic VEGF-2-injected

Diabetic saline-injected

Diabetic VEGF-2-injected

Vessels per cross section

$39.4 \pm 5.8$

$44.1 \pm 7.2^{\mathrm{A}}$

$16.2 \pm 3.4^{\mathrm{B}}$

$48.8 \pm 8.4^{\mathrm{A}, \mathrm{B}}$

Before sacrifice at 8 weeks after treatment (gene transfer or saline-injections), animals were perfused with FITC-conjugated BS-1 lectin to visualize vasa nervorum. Ten cross sections per paraffin-embedded specimen were randomly selected from each specimen, and vessels per cross section were counted under a fluorescence microscope $(\times 4)$ by a blinded investigator. Data are expressed as mean \pm SEM of $n=5$ per study group, where individual numbers per animal were averaged from right and left tibial nerves. ${ }^{A}$ Not significant versus nondiabetic saline-injected. ${ }^{B} P<0.01$ versus nondiabetic saline-injected.
} 


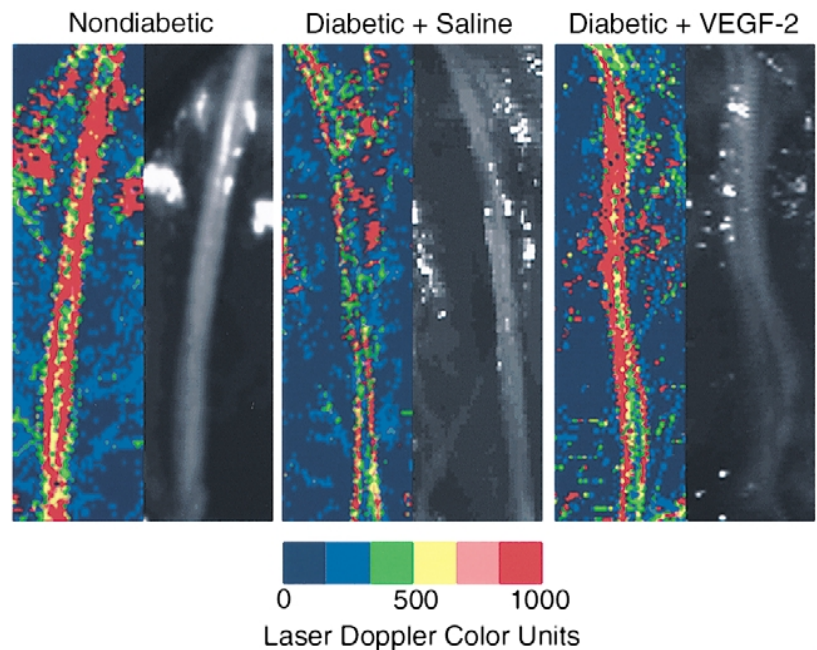

Figure 7

Representative color-coded images of in vivo LDPI in rabbit sciatic nerve 8 weeks after VEGF-2 gene transfer or saline injection. Nerves were surgically exposed from the sciatic notch to the knee level before three repeated LDPI measurements were obtained. Lowest blood flow is indicated in blue, maximum blood flow in red, and intermediate grading in green and yellow. Nondiabetic saline-injected: perfusion of sciatic nerve in a normal, age-matched rabbit that underwent saline injection. Diabetic saline-injected: markedly reduced perfusion of sciatic nerve in a diabetic rabbit, 8 weeks after sham saline injection. Diabetic VEGF-2 gene transfer: substantial restoration of sciatic nerve perfusion in a diabetic rabbit 8 weeks after VEGF gene transfer.

\section{Discussion}

Earlier work from our laboratory (35) used animal models of hindlimb ischemia to document favorable effects of VEGF gene transfer on ischemic peripheral neuropathy. Here, we used two different animal models of experimental non-insulin-dependent and insulin-dependent diabetes that the development of diabetic neuropathy is related to loss of vasa nervorum responsible for perfusion of peripheral nerves. Physiological results were correlated to anatomical observations of the vasa nervorum in the affected nerves of both animal models. In situ fluorescent imaging of whole-mounted explants of sciatic nerves (rats) and tibial portions of the sciatic nerve (rabbits) enabled the assessment of their respective vascularity along their entire length. We found an overall reduction of the number of vasa nervorum in diabetic animals, compared with age-matched nondiabetic controls. VEGF gene transfer restored the vascularization of these nerves to a pattern observed in nondiabetic animals, and this effect was similar for VEGF-1 and VEGF-2 gene transfer. This may explain the mechanism of action of VEGF in this entity. Numerous reports have shown direct effects of reduced blood flow or ischemia on nerve conduction velocity and integrity of the peripheral nervous system $(11-13,36,37)$. In particular, diabetic neuropathy has been causally related to microangiopathy and endoneurial ischemia $(14,15$, 38). Attempts have been made to restore blood flow to the nerve using various agents, such as pentoxifylline (39), iloprost (40), and ACE inhibition (41).

Temporal analysis of electrophysiological findings in the rat model showed that improvements in nerve function became obvious as soon as 2 weeks after gene transfer at a time when transgene expression has been shown to peak (22). At 4 weeks after gene transfer, sciatic nerve MCV and SCV were fully restored, and this effect persisted up to week 10 after treatment. Tailflick testing, a measure for the function of small fiber sensory neurons, demonstrated that the favorable effects of VEGF were not restricted to large fibers and the areas close to the sites of gene transfer, but showed that VEGF-1 gene transfer also improved tailflick temperatures in diabetic rats. Improvement in the results of tailflick testing after VEGF gene transfer contrasts with results seen after administration of recombinant human nerve growth factor (rhNGF) for experimentally induced diabetic neuropathy (10). In that study, rhNGF treatment showed positive effects on small sensory fibers only, but had no effect upon function of large fibers.

To determine further the contribution of the microvasculature to the pathogenesis of diabetic neuropathy, we used a strategy of VEGF gene transfer to preserve and/or restore the integrity of the vasa nervorum. We tested gene transfer of naked DNA plasmids encoding for both VEGF-1 (165-amino acid isoform) and VEGF-2. Electrophysiological examinations showed that both transgenes had the same favorable effects on clinical findings of neuropathy. Although preliminary clinical applications of VEGF gene transfer have indicated that circulating levels of VEGF protein are limited to picogram quantities and for periods
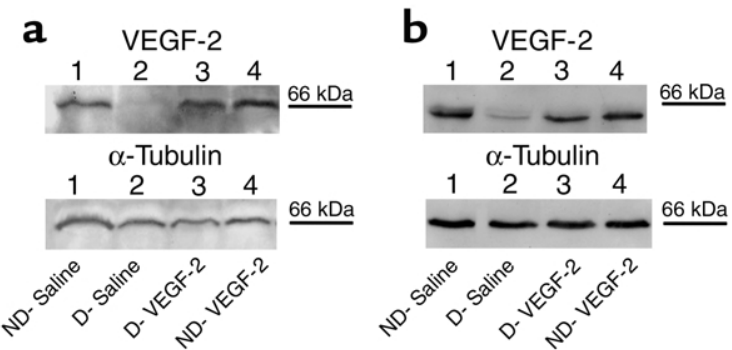

\section{Figure 8}

Expression of VEGF protein in nondiabetic and diabetic ( 6 months after alloxan treatment) rabbits. Western blot analysis of VEGF-2 protein expression in hindlimb muscles (a) and sciatic nerves (b) harvested 2 weeks after sham (saline) or gene injections. Lane 1: nondiabetic, saline injected. Lane 2: diabetic saline-injected. Lane 3: diabetic, VEGF-2 gene transfer. Lane 4: nondiabetic, VEGF-2 gene transfer. Molecular masses are indicated at the right. The precursor form of VEGF-2 is detected as a 58-kDa protein. The level of VEGF-2 protein expression was significantly reduced in diabetic compared with nondiabetic rabbits. VEGF-2 gene transfer led to a restoration of VEGF-2 protein expression in both muscles and nerves of diabetic rabbits that was similar to expression in nondiabetic animals. Western blots were reprobed with an anti- $\alpha$-tubulin antibody to confirm equal protein loading of lanes. Similar results were obtained in four additional experiments. 
of less than 2 weeks after gene transfer, we recognize that administration of an angiogenic growth factor, despite favorable effects in one tissue type, may adversely affect remote tissues such as the kidney and eye (42). We therefore checked renal functional parameters and performed histological analysis of the kidneys and found no evidence of adverse outcomes in either case. Moreover, previous follow-up studies performed by an independent group of retinal specialists who have thus far observed more than 60 patients undergoing VEGF gene transfer for cardiac or lower extremity vascular insufficiency (including diabetics with a previous history of retinopathy) have shown that local delivery of VEGF to ischemic muscle was not associated with development or exacerbation of retinopathy $(43,44)$ in any, including diabetic, subjects (45). These experimental and clinical findings are thus encouraging with regard to these safety issues, although these areas clearly require further investigation and monitoring.

There are currently no therapeutic options for patients with diabetic neuropathy. It is intriguing to note that neurological and neurophysiological findings in a prospective study of 24 patients undergoing phVEGF $_{165}$ gene transfer for critical limb ischemia disclosed clinical improvement and statistically significant improvement in electrophysiological measurements performed in four of the six diabetic patients who completed the study (46). The results of these experiments do not exclude the possible contribution of direct effects of VEGF on nerve integrity $(35,47,48)$. Furthermore, although the animal models used in this study are established models for the study of long-term effects of diabetes (the rats and the rabbits were diabetic for 3 and 6 months, respectively, before gene transfer, resulting in diabetic complications such as cataracts and foot necrosis), the results of experiments used in any animal model do not necessarily reproduce all elements of the human disorder. In particular, whether the neovasculature developing in response to VEGF remains durable within a hyperglycemic milieu, or whether periodic readministration would be required, remains to be clarified.

Nevertheless, the current findings clearly indicate a protective effect of VEGF-induced angiogenesis of vasa nervorum on diabetic neuropathy and thus represent a novel therapeutic paradigm for the treatment of this otherwise irreversible disorder.

\section{Acknowledgments}

This study was supported in part by grants from the NIH (HL57516, HL60911, and HL53354 to J.M. Isner). P. Schratzberger is the recipient of an Erwin Schroedinger fellowship grant, provided by the Austrian Funds for the Advancement of Science.

1. Tomlinson, D.R., Fernyhough, P., and Diemel, L.T. 1997. Role of neurotrophins in diabetic neuropathy and treatment with nerve growth factors. Diabetes. 46:S43-S49.

2. Reiber, G.E., Boyko, E.J., and Smith, D.G. 1995. Lower extremity foot ulcers and amputations in diabetes. In Diabetes in America. M.I. Harris et al., editors. National Institute of Diabetes and Digestive and Kidney Dis- eases. Washington, DC, USA. 409-427.

3. Parkhouse, N., and LeQuesne, P.M. 1988. Impaired neurogenic vascular response in patients with diabetes and neuropathic foot lesions. N. Engl. J. Med. 318:1306-1309.

4. Veves, A., et al. 1998. Endothelial dysfunction and the expression of endothelial nitric oxide synthase in diabetic neuropathy, vascular disease, and foot ulceration. Diabetes. 47:457-463.

5. The Diabetes Control and Complications Trial Research Group. 1993. The effect of intensive treatment of diabetes on the development and progression of long-term complications in insulin-dependent diabetes mellitus. N. Engl. J. Med. 329:977-988.

6. Currie, C.J., Morgan, C.L., and Peters, J.R. 1998. The epidemiology and cost of inpatient care of peripheral vascular disease, infection, neuropathy, and ulceration in diabetes. Diabetes Care. 21:42-48.

7. Cullum, N.A., Mahon, J., Stringer, K., and McLean, W.G. 1991. Glycation of rat sciatic nerve tubulin in experimental diabetes mellitus. Diabetologia. 34:387-389.

8. Greene, D.A., Lattimer, S.A., and Sima, A.F. 1987. Sorbitol, phosphoinositides, and sodium-potassium-ATPase in the pathogenesis of diabetic complications. N. Engl. J. Med. 316:599-606.

9. Cameron, N.E., and Cotter, M.A. 1994. The relationship of vascular changes to metabolic factors in diabetic mellitus and their role in the development of peripheral nerve complications. Diabetes Metab. Rev. 10:189-224.

10. Apfel, S.C., Arezzo, J.C., Brownlee, M., Federoff, H., and Kessler, J.A. 1994. Nerve growth factor administration protects against experimental diabetic sensory neuropathy. Brain Res. 634:7-12.

11. Tuck, R.R., Schmelzer, J.D., and Low, P.A. 1984. Endoneurial blood flow and oxygen tension in the sciatic nerves of rats with experimental diabetic neuropathy. Brain. 107:935-950.

12. Cameron, N.E., Cotter, M.A., and Low, P.A. 1991. Nerve blood flow in early experimental diabetes in rats: relation to conduction deficits. Am. J. Physiol. 261:E1-E8.

13. Tesfaye, S., et al. 1993. Impaired blood flow and arterio-venous shunting in human diabetic neuropathy: a novel technique of nerve photography and fluorescein angiography. Diabetologia. 36:1266-1274.

14. Dyck, P.J. 1989 . Hypoxic neuropathy: does hypoxia play a role in diabetic neuropathy. Neurology. 39:111-118.

15. Stevens, E.J., Carrington, A.L., and Tomlinson, D.R. 1994. Nerve ischemia in diabetic rats: time-course of development, effect of insulin treatment plus comparison of streptozotocin and BB models. Diabetologia. 37:43-48.

16. Leung, D.W., Cachianes, G., Kuang, W.J., Goeddel, D.V., and Ferrara, N. 1989. Vascular endothelial growth factor is a secreted angiogenic mitogen. Science. 246:1306-1309.

17. Dvorak, H.F., Brown, L.F., Detmar, M., and Dvorak, A.M. 1995. Vascular permeability factor/vascular endothelial growth factor, microvascular hyperpermeability, and angiogenesis. Am.J. Pathol. 146:1029-1039.

18. Rivard, A., et al. 1999. Rescue of diabetes related impairment of angiogenesis by intramuscular gene therapy with adeno-VEGF. Am. J. Pathol. 154:355-364.

19. Tsurumi, Y., et al. 1996. Direct intramuscular gene transfer of naked DNA encoding vascular endothelial growth factor augments collateral development and tissue perfusion. Circulation. 94:3281-3290.

20. Rivard, A., et al. 1999. Age-dependent impairment of angiogenesis. Circulation. 99:111-120.

21. Murohara, T., et al. 1998. Nitric oxide synthase modulates angiogenesis in response to tissue ischemia. J. Clin. Invest. 101:2567-2578.

22. Baumgartner, I., et al. 1998. Constitutive expression of phVEGF $_{165}$ following intramuscular gene transfer promotes collateral vessel development in patients with critical limb ischemia. Circulation. 97:1114-1123.

23. Isner, J.M., et al. 1998. Treatment of thromboangiitis obliterans (Buerger's disease) by intramuscular gene transfer of vascular endothelial growth factor: preliminary clinical results. J. Vasc. Surg. 28:964-975.

24. Isner, J.M., et al. 1996. Clinical evidence of angiogenesis following arterial gene transfer of phVEGF ${ }_{165}$. Lancet. 348:370-374.

25. Losordo, D.W., et al. 1998. Gene therapy for myocardial angiogenesis: initial clinical results with direct myocardial injection of phVEGF $_{165}$ as sole therapy for myocardial ischemia. Circulation. 98:2800-2804.

26. Isner, J.M., et al. 1996. Arterial gene transfer for therapeutic angiogenesis in patients with peripheral artery disease. Hum. Gene Ther. 7:959-988.

27. Witzenbichler, B., et al. 1998. Vascular endothelial growth factor-C (VEGF-C/VEGF-2) promotes angiogenesis in the setting of tissue ischemia. Am. J. Pathol. 153:381-394.

28. Joukov, V., et al. 1996. A novel vascular endothelial growth factor, VEGF$\mathrm{C}$, is a ligand for the Flt4 (VEGFR-3) and KDR (VEGFR-2) receptor tyrosine kinases. EMBO J. 15:290-298.

29. Stanley, E.F. 1981. Sensory and motor nerve conduction velocities and the latency of the H reflex during growth in rat. Exp. Neurol. 71:497-506.

30. De Koning, P., and Gispen, W.H. 1987. Org. 2766 improves functional and electrophysiological aspects of regenerating sciatic nerve in the rat. Peptides. 8:415-422. 
31. Couffinhal, T., et al. 1998. A mouse model of angiogenesis. Am. J. Pathol. 152:1667-1679.

32. Alroy, J., Goyal, V., and Skutelsky, E. 1987. Lectin histochemistry of mammalian endothelium. Histochemistry. 86:603-607.

33. Asahara, T., et al. 1998. Tie2 receptor ligands, angiopoietin-1 and angiopoietin-2, modulate VEGF-induced postnatal neovascularization. Circ. Res. 83:233-240.

34. Duh, E., and Aillo, L.P. 1999. Vascular endothelial growth factor and diabetes: the agonist versus antagonist paradox. Diabetes. 48:1899-1906.

35. Schratzberger, P., et al. 2000. Favorable impact of VEGF gene transfer on ischemic peripheral neuropathy. Nat. Med. 6:405-413.

36. Young, M.J., Veves, A., Smith, J.V., Walker, M.G., and Boulton, A.J.M. 1995. Restoring lower limb blood flow improves conduction velocity in diabetic patients. Diabetologia. 38:1051-1054.

37. Tesfaye, S., Harris, R.M., and Ward, J.D. 1992. Exercise-induced conduction velocity increment: a marker of impaired peripheral nerve blood flow in diabetic neuropathy. Diabetologia. 35:155-159.

38. Malik, R.A., et al. 1989. Microangiopathy in human diabetic neuropathy: relationship between capillary abnormalities and the severity of neuropathy. Diabetologia. 32:92-102.

39. Flint, H., Cotter, M.A., and Cameron, N.E. 2000. Pentoxifylline effects on nerve conduction velocity and blood flow in diabetic rats. Int. J. Exp. Diabetes Res. 1:49-58.

40. Cotter, M.A., Dines, K.C., and Cameron, N.E. 1993. Prevention and reversal of motor and sensory peripheral nerve conduction abnormalities in streptozotocin-diabetic rats by the prostacyclin analogue iloprost. Naun- yn Schmiedeberg's Arch. Pharmacol. 347:534-540.

41. Cameron, N.E., Cotter, M.A., and Robertson, S. 1992. Angiotensin converting enzyme inhibition prevents development of muscle and nerve dysfunction and stimulates angiogenesis in streptozotocin-diabetics rats. Diabetologia. 35:12-18.

42. Aiello, L.P. 2000. Keeping in touch with angiogenesis. Nat. Med. 4:379-381.

43. Aiello, L.P., et al. 1994. Vascular endothelial growth factor in ocular fluids of patients with diabetic retinopathy and other retinal disorders. $N$. Engl. J. Med. 331:1480-1487.

44. Adamis, A.P., et al. 1994. Increased vascular endothelial growth factor levels in the vitreous of eyes with proliferative diabetic retinopathy. Am. J. Ophthalmol. 118:445-450.

45. Vale, P.R., Rauh, G., Wuensch, D.I., Pieczek, A., and Schainfeld, R.M. 1998. Influence of vascular endothelial growth factor on diabetic retinopathy. Circulation. 17:I-353. (Abstr.)

46. Simovic, D., Ropper, A.H., Isner, J.M., and Weinberg, D.H. 1999. Improvement in ischemic limb neuropathy after vegf gene transfer. Circulation. 18:I-770. (Abstr.)

47. Sondell, M., Lundborg, G., and Kanje, M. 1999. Vascular endothelial growth factor has neurotrophic activity and stimulates axonal outgrowth, enhancing cell survival and Schwann cell proliferation in the peripheral nervous system. J. Neurol. Sci. 19:5731-5740.

48. Sondell, M., Lundborg, G., and Kanje, M. 1999. Vascular endothelial growth factor stimulates Schwann cell invasion and neovascularization of acellular nerve grafts. Brain Res. 846:219-228. 